\title{
Safety and effectiveness of balloon cryoablation for treatment of Barrett's associated neoplasia: systematic review and meta-analysis
}

\section{다)(1) $\odot(9$}

\author{
Authors \\ Donevan R. Westerveld ${ }^{1}$, Khaai Nguyen ${ }^{2}$, Debdeep Banerjee ${ }^{1}$, Chelsea Jacobs ${ }^{1}$, Nikhil Kadle ${ }^{3}$, Peter V. Draganov ${ }^{3}$, \\ Dennis Yang ${ }^{3}$
}

Institutions

1 Department of Internal Medicine, University of Florida College of Medicine, Gainesville, Florida, United States

2 Khaai Nguyen, College of Agriculture and Life Sciences, University of Florida, Gainesville, Florida, United States

3 Division of Gastroenterology and Hepatology, University of Florida College of Medicine, Gainesville, Florida, United States

submitted 31.7.2019

accepted after revision 17.10.2019

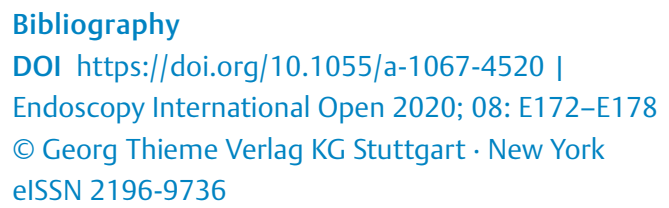

Corresponding author

Dennis Yang, MD, Assistant Professor of Medicine, Division of Gastroenterology, Hepatology, and Nutrition, University of Florida College of Medicine, Gainesville, FL, USA

Fax: +1352-273-7899

dennis.yang@medicine.ufl.edu

Supplemental Table 1

Online content viewable at:

https://doi.org/10.1055/a-1067-4520

\section{ABSTRACT}

Background and study aims Balloon cryoablation (BC) is a novel procedure for endoscopic ablation of Barrett's esophagus (BE- associated neoplasia. We performed a meta-analysis to assess the feasibility, effectiveness, and safety of BC for treatment of BE neoplasia.

Patients and methods Several databases were searched for relevant articles (PubMed, Web of Science, Google Scholar, EMBASE) as well as abstracts of recent gastroenterology meetings. Data extraction was performed by two investigators using standardized forms, including age, gender, length of BE segment, prior treatments, procedural time and number ablation sessions, technical feasibility, adverse events, and eradication rates of intestinal metaplasia (CE-IM) and dysplasia (CE-D) at follow-up. Quality of the studies was assessed using a modified Newcastle Ottawa Scale.

Results Seven studies met inclusion criteria for a total of 548 ablation sessions in 272 patients. The most common histopathology reported prior to BC was high-grade dysplasia $(n=131)$, followed by low-grade dysplasia $(n=75)$, and intramucosal adenocarcinoma $(n=52)$. The pooled rate for technical feasibility was $95.8 \%\left(95 \% \mathrm{Cl}\right.$ : 93.6-97.5\%; $\mathrm{I}^{2}=$ $13.2 \%$; $P=0.3$ ). Pooled rates of CE-IM and CE-D were $85.8 \%$ (95\% Cl: 77.8-92.2\%, $\left.\mathrm{I}^{2}=55.5 \% ; \mathrm{p}=0.04\right)$ and $93.8 \%(95 \%$ $\mathrm{Cl}$ : 85.5-98.7\%, $\left.\mathrm{I}^{2}=74.2 \% ; \quad P=0.001\right)$, respectively. The overall adverse event (AE) rate was $12.5 \%$ (34 out of 272 patients), of which stricture formation was the most common (5.8\%), followed by mucosal laceration $(0.7 \%)$, perforation $(0.4 \%)$, and bleeding $(0.4 \%)$. All AEs were successfully managed endoscopically.

Conclusion This meta-analysis suggests that $B C$ is a safe and effective ablative technique for treatment of $\mathrm{BE}$ neoplasia; future prospective comparative trials are needed to corroborate these initial findings.

\section{Introduction}

Barrett's esophagus (BE) is a metaplastic condition in which squamous epithelium is replaced by specialized intestinal columnar epithelium [1] and is the only known histological precursor to esophageal adenocarcinoma. Multiple endoscopic therapies, including both ablative and resection techniques, have been shown to be safe and effective for BE-associated neoplasia [2-5]. While radiofrequency ablation (RFA) is currently the first-line ablative therapy for treatment of $B E[2,3]$, endoscopic cryotherapy, a technique that induces tissue injury via rapid intracellular freezing [6], has become an attractive alternative. Indeed, cryotherapy has been shown to be effective in achieving complete eradication of intestinal metaplasia (CE- 
IM) and dysplasia (CE-D) in $46 \%$ and $76 \%$ of patients with $B E$ after failed RFA [7].

A novel contact balloon cryoablation (BC) system was recently introduced for management of BE-associated neoplasia (CryoBalloon, Pentax Medical, Redwood City, California, United States) [8]. The new system consists of a portable hand-held device that converts liquid nitrous oxide into its gaseous form within a contact balloon system [8]. Potential advantages of $\mathrm{BC}$ include its portability, ease of use, consistent cryogen dosing delivery to the target site, and lower costs when compared to other existing ablation systems [9]. Furthermore, the new system does not require any capital equipment acquisition which may facilitate usability and introduction in various practice settings. Utilizing this novel technologic intervention should lead to high rates of CE-IM and CE-D in patients with $B E$. The aim of this study was to determine the safety and effectiveness of $\mathrm{BC}$ for the treatment of $\mathrm{BE}$ by performing a meta-analysis of the available literature.

\section{Patients and methods}

\section{Search strategy}

We identified studies by performing a systematic literature search of electronic databases (PubMed, Web of Science, Google Scholar, EMBASE) from their inception through June 2019, with the assistance of a medical librarian. Searches were performed using a combination of the following terms: C2 cryoballoon*, cryoballoon ablation barrett*, barrett cryotherapy*, cryoballoon ablation esophagus*, cryoballoon ablation device*, barrett esophagus, cryoablat*, cryo*, freeze*, cryoballoon focal*, focal cryoablation*. The search query results were reviewed by three investigators (DRW, NK, DY) for relevance based on the pre-established inclusion and exclusion criteria. The full-text of the pertinent studies were then reviewed by the investigators for data extraction and analysis. We attempted to identify any additional studies by also reviewing the bibliographies of the included studies and by performing a manual search to retrieve other potentially relevant studies that may have been omitted on our initial search strategy. Any discrepancies were resolved by consensus and/or by consulting a fourth investigator (PVD).

\section{Study selection}

Three investigators (DRW, NK, DY) screened all titles and abstracts for studies reporting outcomes of CryoBalloon ablation in $\mathrm{BE}$. The articles were reviewed in full text and included in the meta-analysis if they met the following pre-defined inclusion criteria: (1) studies included at least five patients with BE treated with cryotherapy via CryoBalloon device, (2) studies reported the rate of CE-IM and/or CE-D after at least one cryotherapy treatment session. Studies were excluded if: (1) there were less than five patients included in the study; (2) cryotherapy was performed with a device other than the balloon-based liquid nitrous oxide; (3) CE-IM or CE-D could not be determined; (4) the study was a commentary, review, or survey; (5) duplicate studies; or (6) publication was in a language other than English.

\section{Data extraction and quality assessment}

Two investigators (DRW, DY) independently extracted data from each study by using a standardized abstraction form. The standardized data abstraction form included: (1) authors; (2) year of publication; (3) setting; (4) study design; (5) number of patients and baseline characteristics; (6) length of BE segment; (7) prior treatments (e.g. RFA, EMR); (8) procedure characteristics (procedure time, number of ablation sessions), (9) adverse events (AEs); (10) rate of CE-IM and CE-D; and (11) follow-up period.

Quality of the studies was formally evaluated by two investigators (DRW, DY) by using a modified Newcastle Ottawa Scale (NOS) for cohort studies as previously described [7, 10], consisting of six components, each valued up to 1 point: cohort size, length of BE segment, histopathology prior to cryotherapy, endoscopic therapy prior to cryotherapy, number of cryotherapy sessions before assessment of outcomes, and adequacy of follow-up. Studies with scores $>3$ or $\leq 3$ were considered to be of high or low quality, respectively. Funnel plots were generated to evaluate for publication bias [11]. Any discrepancies were resolved by input by a third investigator (PVD) with general consensus among all of the investigators.

\section{Outcome measures and data analysis}

The primary aims of this study were to identify the rate of CE-IM an CE-D among patients with BE after cryotherapy with the balloon-based liquid nitrous oxide device (CryoBalloon). Secondary outcomes included technical feasibility and rate of adverse events. Technical feasibility was defined as the ability to complete the ablation session as intended. AEs included in the standardized abstraction form included perforation, mucosal laceration/injury, bleeding, and stricture formation.

\section{Statistical analysis}

This study was performed in accordance with the criteria established in the Preferred Reporting Items for Systematic Reviews and Meta-Analyses (PRISMA) and Meta-analyses of Observational studies in Epidemiology (MOOSE) checklists [12,13]. Pooled proportions for the outcome measures were calculated using a random effects model to account for differences in study size. The Cochran $\mathrm{Q}$ test and $\mathrm{I}^{2}$ were used to assess for heterogeneity among the included studies. $I^{2}$ values of $<25 \%$, $25 \%$ to $50 \%$ and $>50 \%$ were considered to represent low, moderate and high heterogeneity, respectively. $P<0.05$ was considered significant and all tests were two-tailed. Prediction intervals for the net effect were calculated for feasibility, CE-IM, and CE-D [14]. Analysis was conducted using Stata, version 15 (Stata Corp, College Station, Texas, United States).

\section{Results}

\section{Search results}

Our search strategy yielded 1407 studies, of which 604 were duplicates. Of the remaining 803 studies, 793 were deemed irrelevant based on title and abstract review. Full review was subsequently performed on 11 studies. Of these, three case re- 
ports ( $<5$ patients) were excluded [15-17]; and one study was excluded because it reported outcomes of CryoBalloon ablation in squamous cell cancer of the esophagus [18]. The remaining seven studies fulfilled the predefined inclusion and exclusion criteria for meta-analysis ( Fig. 1) [9, 19-24].

\section{Study characteristics and quality assessment}

All studies were observational, from the United States and the Netherlands, and published between 2015 to 2019 ( $\triangleright$ Table 1). Most studies were single-center in design $(n=5)$. Three studies were published in abstract form as compared to four studies published in full text. The seven studies cumulatively reported outcomes in 272 patients with BE treated with CryoBalloon ablation. Most patients were men (198/272; 72.8\%) and older. The Prague classification was provided in 5 studies; with 3 studies reporting mean and/or median lengths of BE consistent with long-segment $B E(\geq 3 \mathrm{~cm})$. Two studies excluded patients with prior RFA treatment $[19,24]$, three studies included patients with prior RFA $[9,22,23]$, whereas status of RFA was not specified in the remainder two studies $[20,21]$. Most studies $(n=5)$ reported whether EMR was performed as part of the management of BE patients with visible lesions. In aggregate, the most common histopathology reported prior to CryoBalloon ablation was high-grade dysplasia $(n=131)$, followed by low-grade dysplasia $(n=75)$, and intramucosal adenocarcinoma $(n=52)$. Ablation was reported in nine patients with no dysplasia and in one patient with indefinite dysplasia. Liquid nitrogen application duration of 10 seconds was utilized in all studies except one [23], which also included ablation durations of 6 and 8 seconds. In five of seven studies, outcomes were assessed following a single ablation session.

Quality assessment of the included studies were evaluated by using the modified NOS scale as shown in $>$ Table 2. Overall, five studies were found to be of high quality and two studies were deemed low quality based on our scale (Supplemental Table 1).

\section{Main outcomes}

\section{Technical feasibility}

The seven studies cumulatively reported 548 ablation sessions in 272 patients. The pooled rate of technical success, defined as completion of the ablation procedure as intended, was $95.8 \%$ ( $95 \% \mathrm{Cl}$ : 93.6-97.5\%; $P=0.3$ ) with $\mathrm{I}^{2}=13.2 \%$ in the random effects model ( $\mathbf{F i g . 2 a )}$ ). The $95 \%$ prediction interval was calculated to be $0.85-0.95$. Reasons for failed cryoablation included: difficult positioning of the balloon at the gastroesophageal junction $(n=2)[19,22]$, pre-existing stricture $(n=3)[9,19$, 21], ablation of non-BE mucosa $(n=1)$ [19], and device failure $(n=3)$ [19]. There was no evidence of substantial publication bias based on visual inspection of the funnel plot ( $\mathbf{F i g . 3 a ) . ~}$

\section{Complete eradication of intestinal metaplasia (CE-IM) and dysplasia (CE-D)}

Among the seven studies consisting of 272 patients, CE-IM was reported in 228 following cryotherapy. The pooled proportion of CE-IM was $85.8 \%$ (95\% Cl: 77.8-92.2\%; $P=0.04)$ with high

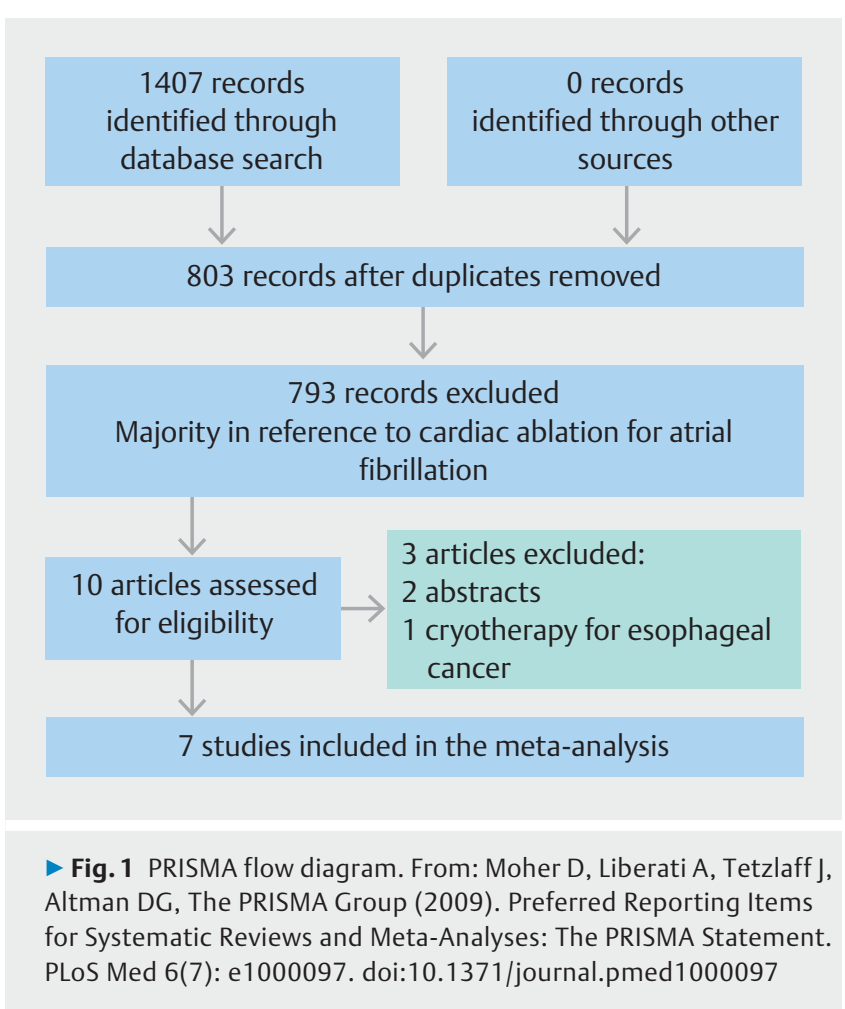

degree of heterogeneity $\left(I^{2}=55.5 \%\right)$ ( $\boldsymbol{F}$ Fig. 2 b) and a $95 \%$ prediction interval of $0.77-0.95$. When the two low-quality studies $[20,21]$ were excluded, the pooled proportion of CE-IM was $87.7 \%$ (95\% Cl: 81.8-92.7\%; $P=0.18$ ) with moderate heterogeneity $\left(I^{2}=36.2 \%\right)$.

There were a total of 262 patients with dysplastic BE among the seven studies included in the meta-analysis. CE-D was reported in 238 of these patients following cryotherapy. The pooled proportion of CE-D was $93.8 \%$ (95\% Cl: 85.5-98.7\%; $P=$ 0.001 ) with $\mathrm{I}^{2}=74.2 \%$ using a random-effects model ( $\boldsymbol{F}$ Fig. $2 \mathbf{c}$ ). The $95 \%$ prediction interval was $0.84-1.04$. When the two lowquality studies $[20,21]$ were excluded, the pooled proportion of CE-D was 93.1\% (95\% Cl: 82.4-99.0; $P<0.001)$ with a still high degree of heterogeneity among the studies $\left(I^{2}=82.8 \%\right)$.

There was no evidence of substantial publication bias on the pooled proportions of CE-IM and CE-D on visual inspection of the funnel plots ( $\triangleright$ Fig. 3 b and $\triangleright$ Fig. 3c).

\section{Adverse events}

All studies reported post-procedural AEs. Among the seven studies in which 272 patients underwent BC, a total of $34 \mathrm{AEs}$ (12.5\% of patients) were reported. In aggregate, there were 16 cases of post-ablation stricture formation (5.8\%), two severe cases of mucosal laceration $(0.7 \%)$, one case of perforation $(0.4 \%)$ and one case of gastrointestinal bleeding $(0.4 \%)$.

\section{Discussion}

Cryotherapy has emerged as an endoscopic ablative option for patients with BE-associated neoplasia. Recently, a contact balloon cryotherapy device (CryoBalloon; Pentax Medical, Red- 


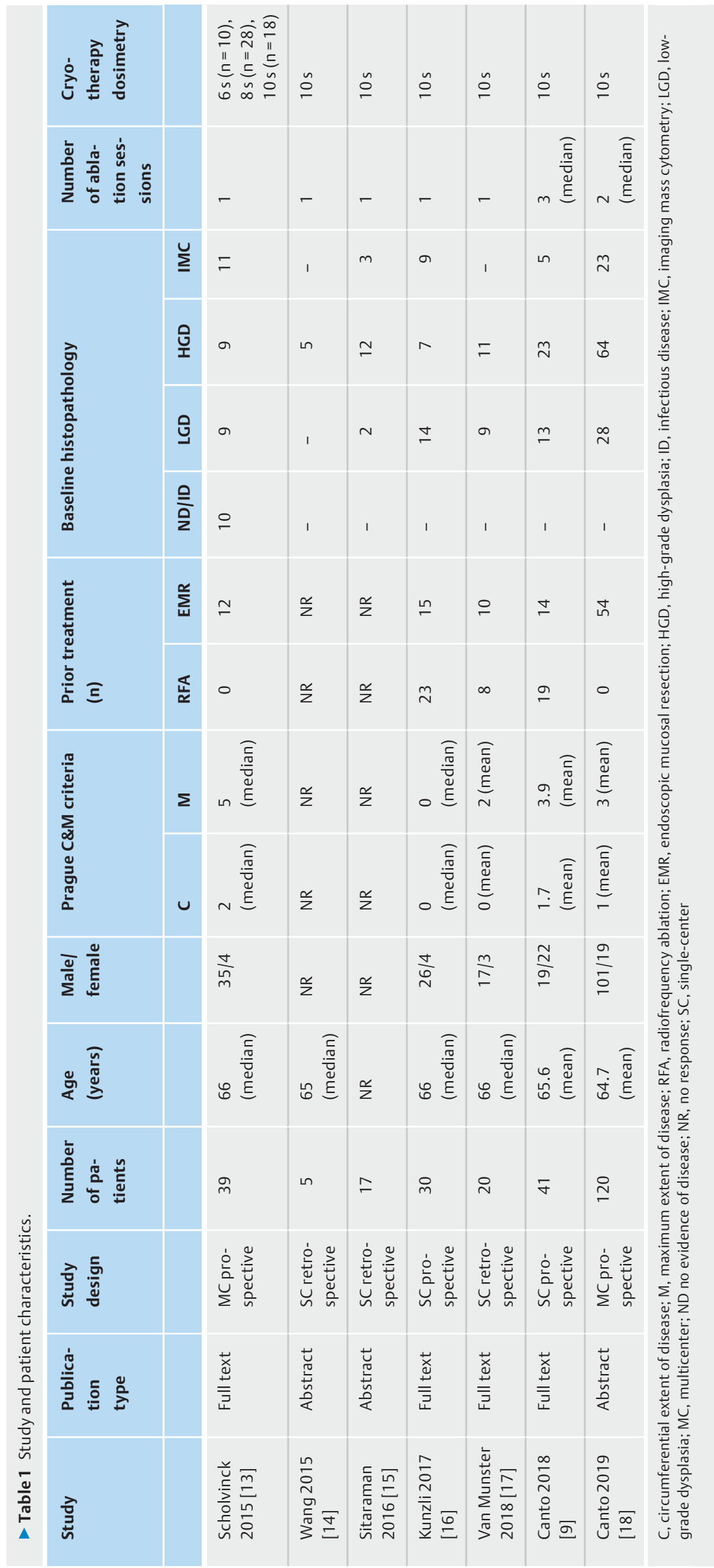


- Table2 Assessment of study quality with modified Newcastle-Ottawa Scale.

\begin{tabular}{|c|c|c|c|c|c|c|c|}
\hline Study & $\begin{array}{l}\text { Large co- } \\
\text { hort size }\end{array}$ & $\begin{array}{l}\text { Length of BE } \\
\text { segment }\end{array}$ & $\begin{array}{l}\text { Baseline histo- } \\
\text { pathology }\end{array}$ & $\begin{array}{l}\text { RFA/EMR prior } \\
\text { to cryotherapy }\end{array}$ & $\begin{array}{l}\text { No. cryotherapy ses- } \\
\text { sions/dosimetry }\end{array}$ & $\begin{array}{l}\text { Adequacy of } \\
\text { follow-Up }\end{array}$ & Total \\
\hline Scholvinck 2015 [13] & 1 & 1 & 1 & 1 & 0 & 1 & 5 \\
\hline Wang 2015 [14] & 0 & 0 & 1 & 0 & 1 & 0 & 2 \\
\hline Sitaraman 2016 [15] & 0 & 0 & 1 & 0 & 1 & 0 & 2 \\
\hline Kunzli 2017 [16] & 1 & 1 & 1 & 1 & 1 & 1 & 6 \\
\hline Van Munster 2018 [17] & 0 & 1 & 1 & 1 & 1 & 1 & 5 \\
\hline Canto 2018 [9] & 1 & 1 & 1 & 1 & 1 & 1 & 6 \\
\hline Canto 2019 [18] & 1 & 1 & 1 & 1 & 1 & 0 & 5 \\
\hline
\end{tabular}

wood City, California, United States) was introduced [8]. Our meta-analysis, which included seven studies comprising 272 patients with $B E$, demonstrated that $B C$ was successful in achieving CE-IM in $88 \%$ and CE-D in $94 \%$ of patients.

Liquid nitrogen spray type (CSA Medical, Lexington, Massachusetts, United States) has been the most widely studied form of cryotherapy delivery method, as compared to the more recent BC. The majority of studies on spray cryotherapy have largely been retrospective, single-arm prospective or registry studies [25-29]. These studies suggest that spray cryotherapy may achieve similar CE-IM and CE-D rates when compared to RFA, and that cryotherapy is an effective option in those who have failed to respond to RFA [7, 30]. From a technical standpoint, spray cryotherapy facilitates treatment of large areas, but visualization for targeted therapy can be hindered by the cryogen spray obscuring the endoscopic lens. Furthermore, the gastrointestinal lumen must be intermittently decompressed with an external tube to prevent excess gas accumulation during treatment. Lastly, the device requires capital investment, which must be factored from a cost perspective.

The $\mathrm{BC}$ is a portable device that is self-venting, as the cryogen spray is contained within the balloon that contacts the targeted area in the esophagus. Our meta-analysis demonstrated that ablation with BC was successfully performed in $96 \%$ of patients, even though most of the included studies used the earlier version of the system. Since then, next-generation CryoBalloon devices have been introduced, including the addition of a pear-shaped balloon to assist with contact treatment at the gastroesophageal junction and wider field cryogen probes to target larger areas. We can only speculate that these next-generation CryoBalloon systems will further enhance ease of use and widen its application [31], still, further data are needed on both technique and optimal dosing strategies.

The primary aim of this meta-analysis was to evaluate the effectiveness of BC for treatment of patients with BE. Our study demonstrated that $\mathrm{BC}$ was associated with high rates of CE-IM (88\%) and CE-D (94\%). We calculated the $95 \%$ prediction intervals to evaluate the variability of the effect of ablation that are likely to be seen in other patients. The interval for CE-IM $0.77-$ 0.95 shows a narrow and favorable range of effect in future po- tential patients. Because of a higher between study heterogeneity, the prediction interval for CE-D was found to be $0.84-$ 1.04. This range covering the null effect again underscores the need for further larger studies. Given the limited literature and relatively small number of patients, we included all studies involving $B C$ for patients with $B E$. This strategy may have introduced heterogeneity as both treatment naïve and RFA non-responders were included in the analysis. Nonetheless, our findings suggest that $B C$ is highly effective for the treatment of $B E$, even when including cohort of patients who have previously failed RFA. Future larger studies are needed to better define the role of $\mathrm{BC}$ as a primary or secondary endoscopic ablative technique in BE associated neoplasia.

Our study suggests that BC is associated with an acceptable safety profile, with an overall $A E$ rate of $12.5 \%$. In this meta-analysis, post-procedural stricture formation was noted in $5.8 \%$ of patients, which is comparable with the $5 \%$ esophageal structuring rate reported after RFA [2]. Furthermore, there were only two cases of serious AEs (1 perforation and 1 Gl bleeding) reported with BC. Overall, our meta-analysis suggests that the post-procedural AEs with BC are similar to those for both RFA and spray cryotherapy [2,27-29].

We acknowledge the limitations of this meta-analysis. Given the relative novelty of $\mathrm{BC}$ for the treatment of $\mathrm{BE}$, all of the included studies were relatively small, observational, and many had only been published in abstract form alone. As previously alluded, the pooled proportions of CE-IM and CE-D included both treatment naïve and RFA refractory patients with BE, which contributed to the heterogeneity noted in the analysis and the possibility of confounding of the effectiveness outcome. Moreover, conducting a meta-analysis of observational studies presents challenges in interpreting the study outcome due to the inherent biases in the included studies. In this study confounding biases may include the aforementioned lack of reporting of patient treatment with RFA in two studies and reporting of treatment duration to name a few. Publication bias, as in the selection of publications based on their cohort size and language of publication should also be considered. This study reports funnel plots to aid in detection of publication bias, how- 


\section{Study}

Percent

ID

(95\% Cl)

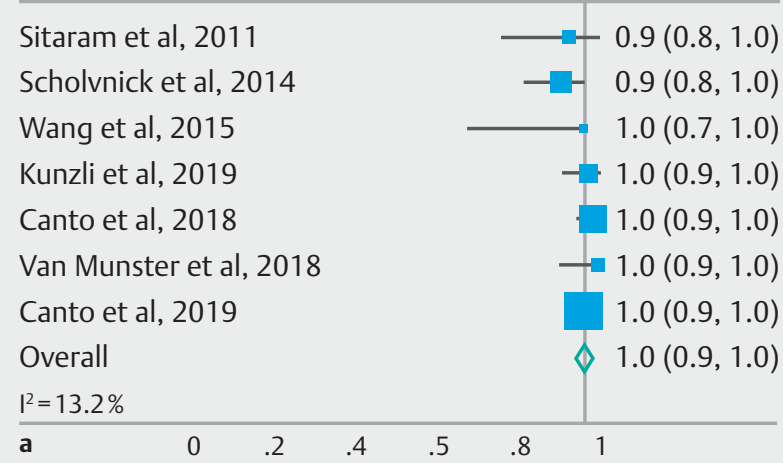

\section{Study}

Percent

ID

$(95 \% \mathrm{Cl})$

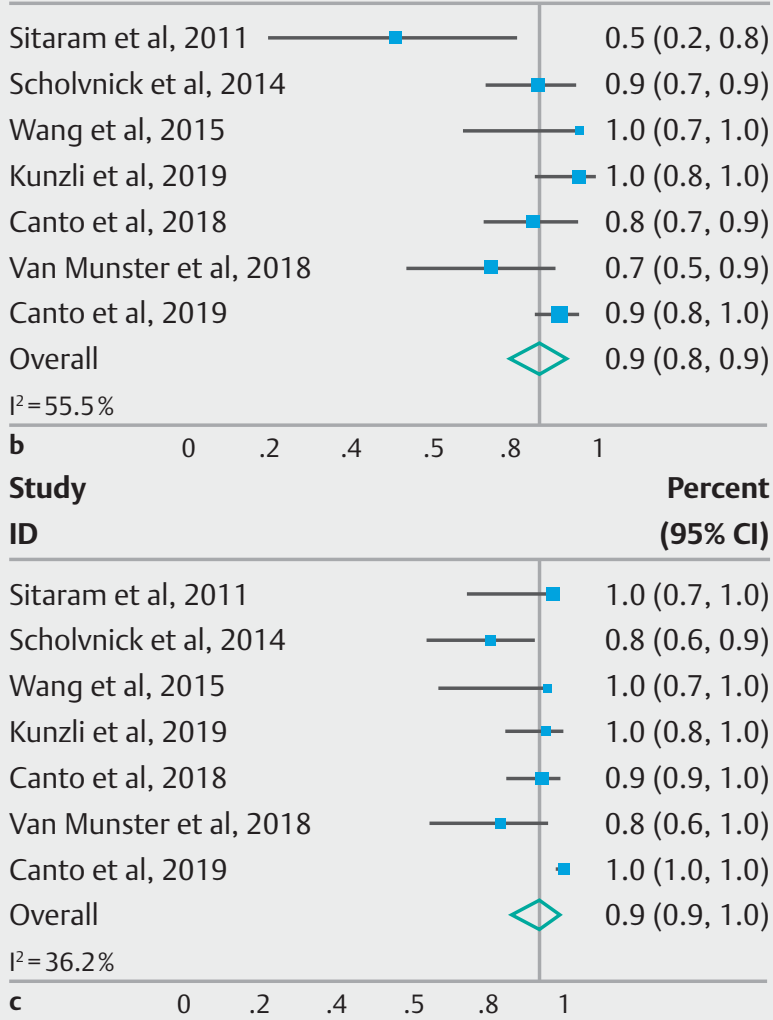

- Fig. 2 a Forest plots of the included studies evaluating the feasibility of CDFAS. b CE-IM rate, and c CE-D rate. CI, confidence interval; overall, overall effect size; random effect model; CE-IM, complete eradication of intestinal metaplasia; CE-D, complete eradication of dysplasia.

ever, the lack of sensitivity analysis for confounders presents a limitation of this study [13].

Furthermore, many of the studies evaluated clinical outcomes following only one session of BC. The relatively short follow-up precludes any definitive conclusions on the effectiveness of BC in maintaining CE-IM and CE-D and the potential rate of incomplete treatment vs recurrence rates. Nonetheless,
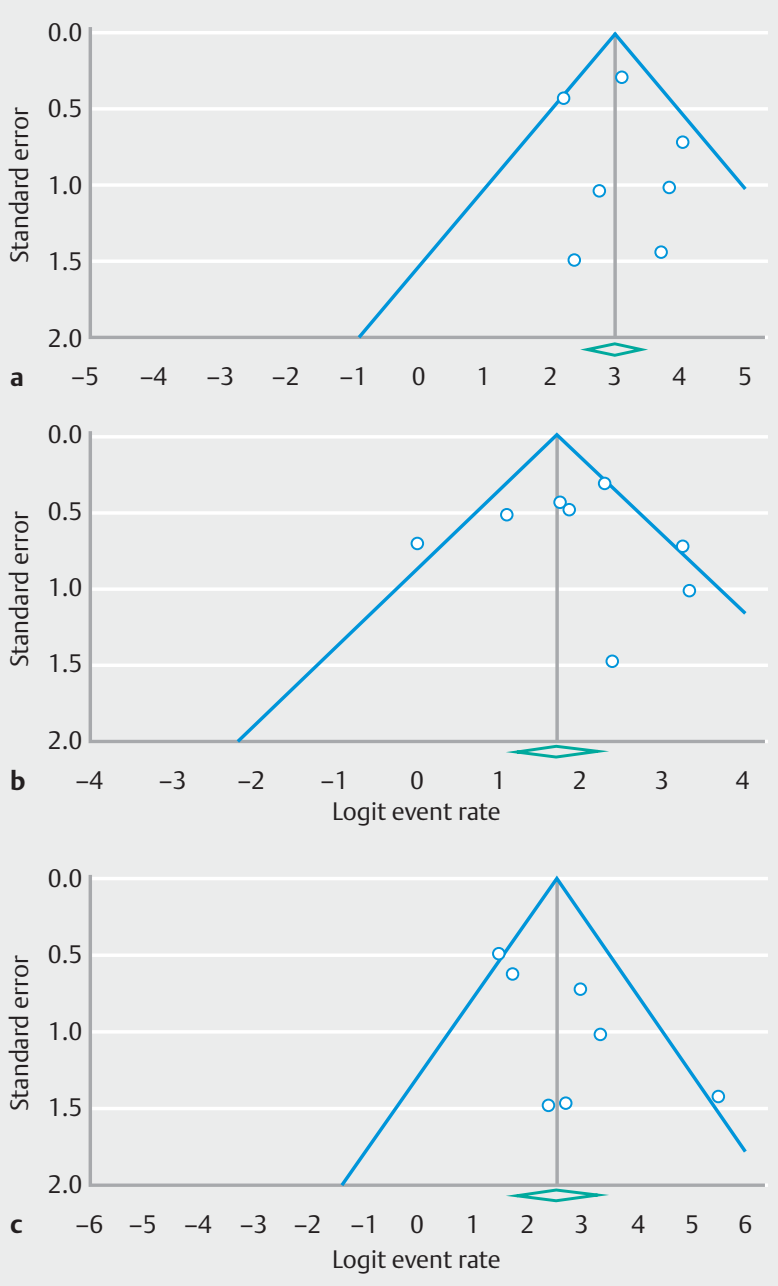

- Fig. 3 a Funnel plots calculated to indicate publication bias for the feasibility of performing CDFAS, b CE-IM, and c CE-D. CDFAS, cryoballoon focal ablation system; CE-IM, complete eradication of intestinal metaplasia; CE-D, complete eradication of dysplasia.

this meta-analysis of the current available literature on $B C$ is promising, albeit underscores the need for high-quality studies.

\section{Conclusion}

In summary, this meta-analysis suggests that $B C$ is technically feasible and associated with high rates of both CE-IM and CE-D in patients with $B E$. BC has a similar safety profile to both spray cryotherapy and RFA, with stricture formation encountered in approximately $6 \%$ of patients. The cryoablation balloon is a novel, portable, easy-to-use device that may be suitable for primary and secondary treatment of patients with BE. Larger prospective, and preferably comparative trials are needed to further define the role of $\mathrm{BC}$ in the treatment algorithm of patients with $\mathrm{BE}$-associated neoplasia. 


\section{Acknowledgements}

The authors would like to thank Dr. Rebecca Beyth for her assistance in statistical analysis.

\section{Competing interests}

Dennis Yang is a consultant for Boston Scientific, Lumendi, and Steris. Peter V. Draganov is a consultant for Boston Scientific, Olympus, Cook Medical, Lumendi and Microtech.

\section{References}

[1] de Jonge PJ, van Blankenstein M, Looman CW et al. Risk of malignant progression in patients with Barrett's oesophagus: a Dutch nationwide cohort study. Gut 2010; 59: 1030-1036

[2] Orman ES, Li N, Shaheen NJ. Efficacy and durability of radiofrequency ablation for Barrett's Esophagus: systematic review and meta-analysis. Clin Gastroenterol Hepatol 2013; 11: 1245-1255

[3] Phoa KN, Van Vilsteren FG, Weusten BL et al. Radiofrequency ablation vs endoscopic surveillance for patients with Barrett esophagus and low-grade dysplasia: a randomized clinical trial. JAMA 2014; 31: 1209-1217

[4] Yang D, Othman M, Draganov PV. Endoscopic Mucosal Resection vs. Endoscopic submucosal dissection for Barrett's esophagus and colorectal neoplasia. Clin Gastroenterol Hepatol 2018; 3: 1021-1028

[5] Yang D, Zou F, Xiong S et al. Endoscopic submucosal dissection for early Barrett's neoplasia: a meta-analysis. Gastrointest Endosc 2018; 87: 1383-1393

[6] Johnston CM, Schoenfeld LP, Ysore JV et al. Endoscopic spray cryotherapy: a new technique for mucosal ablation in the esophagus. Gastrointest Endosc 1999; 50: 86-92

[7] Visrodia K, Zakko L, Singh S et al. Cryotherapy for persistent Barrett's esophagus after radiofrequency ablation: a systematic review and meta-analysis. Gastrointest Endosc 2018; 87: 1396-1404

[8] Friedland S, Triadafilopoulos G. A novel device for ablation of abnormal esophageal mucosa (with video). Gastrointest Endosc 2011; 74: 182-188

[9] Canto MI, Shaheen NJ, Almario JA et al. Multifocal nitrous oxide cryoballoon ablation with or without EMR for treatment of neoplastic Barrett's esophagus (with video). Gastrointest Endosc 2018; 88: 438446

[10] Stang A. Critical evaluation of the Newcastle-Ottawa scale for the assessment of the quality of nonrandomized studies in meta-analyses. Eur J Epidemiol 2010; 25: 603-605

[11] Derry S, Loke YK, Aronson JK. Incomplete evidence: the inadequacy of databases in tracing published adverse drug reactions in clinical trials. BMC Med Res Methodol 2001; 1: 7

[12] Moher D, Liberati A, Tetzlaff J et al. Preferred reporting items for systematic reviews and meta-analyses: the PRISMA statement. Ann Intern Med 2009; 18: 264-269

[13] Stroup DF, Berlin JA, Morton SC et al. Meta-analysis of observational studies in epidemiology: a proposal for reporting; meta-analysis of observational studies in epidemiology (MOOSE) group. JAMA 2000; 15: 2008-2012
[14] Riley RD, Higgins JPT, Deeks J]. Interpretation of random effects meta-analyses. BMJ 2011; 10: 342-349

[15] Trindade AJ, Canto MI. Circumferential treatment of long-segment Barrett's esophagus using the next-generation cryoballoon. Endoscopy 2019; 4: E69-E70

[16] Castela J, Demedts I, Bisschops R. Treatment of low grade dysplasia in Barrett's esophagus with a new-generation cryoballoon device. Endoscopy 2018; 11: E318-E319

[17] Louie BE, Hofstetter W, Triadafilopoulos G et al. Evaluation of a novel cryoballoon swipe ablation system in bench, porcine, and human esophagus models. Dis Esophagus 2018; 8: 1-7

[18] Canto MI, Abrams JA, Kunzli HT et al. Nitrous oxide cryotherapy for treatment of esophageal squamous cell neoplasia: initial multicenter international experience with a novel cryoballoon ablation system (with video). Gastrointest Endosc 2018; 2: 574-581

[19] Schölvinck DW, Künzli HT, Kestens C et al. Treatment of Barrett's esophagus with a novel focal cryoablation device: a safety and feasibility study. Endoscopy 2015; 47: 1106-1112

[20] Wang KK, Chen AM, Friedland S et al. Tu1592 Multi-Center Experience With the Cryoballoon Focal Ablation System (CAS) for Esophageal Dysplasia. Gastrointest Endosc 2015; 81: AB522

[21] Sitaraman L, Lightdale C], Abrams JA. Use of the Cryoballoon focal ablation system for the eradication of esophageal neoplasia: a singlecenter experience. Gastroenterology 2016; 150: S266

[22] Künzli HT, Schölvinck DW, Meijer SL et al. Efficacy of the CryoBalloon Focal Ablation System for the eradication of dysplastic Barrett's esophagus islands. Endoscopy 2017; 49: 169-175

[23] van Munster SN, Overwater A, Haidry R et al. Focal cryoballoon versus radiofrequency ablation of dysplastic Barrett's esophagus: impact on treatment response and postprocedural pain. Gastrointest Endosc 2018; 88: 795-803

[24] Canto MI, Trindade AJ, Abrams J et al. Safety and efficacy of multifocal cryoballoon ablation for eradiction of previously untreated Barrett's neoplasia: preliminary results of a large multicenter American trial. Gastrointest Endsoc 2019; 89: AB98

[25] Suchniak-Mussari K, Dye CE, Moyer MT et al. Efficacy and safety of liquid nitrogen cryotherapy for treatment of Barrett's esophagus. World J Gastrointest Endosc 2017; 16: 480-485

[26] Trindade AJ, Pleskow DK, Sengupta $\mathrm{N}$ et al. Efficacy of liquid nitrogen cryotherapy for Barrett's esophagus after endoscopic resection of intramucosal cancer: a multicenter study. J Gastroenterol Hepatol 2018; 33: 461-465

[27] Shaheen NJ, Greenwald BD, Peery AF et al. Safety and efficacy of endoscopic spray cryotherapy for Barrett's esophagus with highgrade dysplasia. Gastrointest Endosc 2010; 71: 680-685

[28] Ghorbani S, Tsai FC, Greenwald BD et al. Safety and efficacy of endoscopic spray cryotherapy for Barrett's dysplasia: results of the National Cryospray Registry. Dis Esophagus 2016; 29: 241-247

[29] Dumot JA, Vargo JJ, Falk GW et al. An open-label, prospective trial of cryospray ablation for Barrett's esophagus high-grade dysplasia and early esophageal cancer in high-risk patients. Gastrointest Endosc 2009; 70: 635-644

[30] Cholapranee A, Trindade A]. Challenges in endoscopic therapy of dysplastic Barrett's esophagus. Curr Treat Options Gastroenterol 2019; 17: 32-47

[31] Trindade AJ, Canto MI. Circumferential treatment of long-segment Barrett's esophagus using the next-generation cryoballoon. Endoscopy 2019; 51: E69-E70 